PROCEEDINGS OF THE

AMERICAN MATHEMATICAL SOCIETY

Volume 126, Number 11, November 1998, Pages 3315-3327

S 0002-9939(98)04445-1

\title{
PLANCHEREL-PÔLYA TYPE INEQUALITY ON SPACES OF HOMOGENEOUS TYPE AND ITS APPLICATIONS
}

\author{
Y.-S. HAN
}

(Communicated by J. Marshall Ash)

\begin{abstract}
In this paper, using the discrete Calderon reproducing formula on spaces of homogeneous type obtained by the author, we obtain the PlancherelPôlya type inequalities on spaces of homogeneous type. These inequalities give new characterizations of the Besov spaces $\dot{B}_{p}^{\alpha, q}$ and the Triebel-Lizorkin spaces $\dot{F}_{p}^{\alpha, q}$ on spaces of homogeneous type introduced earlier by the author and E. T. Sawyer and also allow us to generalize these spaces to the case where $p, q \leq 1$. Moreover, using these inequalities, we can easily show that the Littlewood-Paley $G$-function and $S$-function are equivalent on spaces of homogeneous type, which gives a new characterization of the Hardy spaces on spaces of homogeneous type introduced by Macias and Segovia.
\end{abstract}

\section{INTRODUCTION}

We begin by recalling the definitions necessary for introducing the Besov and Triebel-Lizorkin spaces on spaces of homogeneous type. A quasi-metric $d$ on a set $X$ is a function $d: X \times X \rightarrow[0, \infty)$ satisfying:

(i) $d(x, y)=0$ if and only if $x=y$,

(ii) $d(x, y)=d(y, x) \quad$ for all $x, y \in X$,

(iii) There exists a constant $A<\infty$ such that for all $x, y$, and $z \in X$,

$$
d(x, y) \leq A[d(x, z)+d(z, y)] .
$$

Any quasi-metric defines a topology, for which the balls $B(x, r)=\{y \in X: d(y, x)$ $<r\}$ form a base. However, the balls themselves need not be open when $A>1$.

Definition $1.2([\mathrm{CW}])$. A space of homogeneous type $(X, d, \mu)$ is a set $X$ together with a quasi-metric $d$ and a nonnegative measure $\mu$ on $X$ such that $\mu(B(x, r))<\infty$ for all $x \in X$ and all $r>0$, and there exists $A^{\prime}<\infty$ such that for all $x \in X$ and all $r>0$,

$$
\mu(B(x, 2 r)) \leq A^{\prime} \mu(B(x, r)) .
$$

Here $\mu$ is assumed to be defined on a $\sigma$-algebra which contains all Borel sets and all balls $B(x, r)$.

Received by the editors September 19, 1996 and, in revised form, April 1, 1997.

1991 Mathematics Subject Classification. Primary 42B25, 46F05.

Key words and phrases. Plancherel-Pôlya type inequality, spaces of homogeneous type, Besov and Triebel-Lizorkin spaces, Littlewood-Paley $G$-function and $S$-function, discrete Calderon formula. 
Macias and Segovia [MS1] have shown that one can replace $d$ by another quasi metric $\rho$ such that there exist $c<\infty$ and some $\theta, 0<\theta<1$, such that

$$
\rho(x, y) \approx \inf \{\mu(B): B \text { is a ball containing } x \text { and } y\},
$$

$$
\left|\rho(x, y)-\rho\left(x^{\prime}, y\right)\right| \leq c \rho\left(x, x^{\prime}\right)^{\theta}\left[\rho(x, y)+\rho\left(x^{\prime}, y\right)\right]^{1-\theta} \quad \text { for all } x, x^{\prime} \text { and } y \in X .
$$

We will suppose that $\mu(X)=\infty$ and $\mu(\{x\})=0$ for all $x \in X$. These hypotheses allow us to construct an approximation to the identity (see [HS] and [H2]).

Definition 1.6. A sequence $\left(S_{k}\right)_{k \in \mathbb{Z}}$ of operators is called an approximation to the identity if $S_{k}(x, y)$, the kernels of $S_{k}$, are functions from $X \times X$ into $\mathbb{C}$ such that there exists a constant $C$ for all $k \in \mathbb{Z}$, for all $x, x^{\prime}, y$ and $y^{\prime} \in X$, and for some $0<\varepsilon \leq \theta$ and $c<\infty$,

(i)

$$
\left|S_{k}(x, y)\right| \leq c \frac{2^{-k \varepsilon}}{\left(2^{-k}+\rho(x, y)\right)^{1+\varepsilon}}
$$

(ii)

$$
\left|S_{k}(x, y)-S_{k}\left(x^{\prime}, y\right)\right| \leq c\left(\frac{\rho\left(x, x^{\prime}\right)}{2^{-k}+\rho(x, y)}\right)^{\varepsilon} \frac{2^{-k \varepsilon}}{\left(2^{-k}+\rho(x, y)\right)^{1+\varepsilon}}
$$

for $\rho\left(x, x^{\prime}\right) \leq \frac{1}{2 A}\left(2^{-k}+\rho(x, y)\right)$;

(iii)

$$
\left|S_{k}(x, y)-S_{k}\left(x, y^{\prime}\right)\right| \leq c\left(\frac{\rho\left(y, y^{\prime}\right)}{2^{-k}+\rho(x, y)}\right)^{\varepsilon} \frac{2^{-k \varepsilon}}{\left(2^{-k}+\rho(x, y)\right)^{1+\varepsilon}}
$$

for $\rho\left(y, y^{\prime}\right) \leq \frac{1}{2 A}\left(2^{-k}+\rho(x, y)\right)$;

(iv)

$$
\begin{aligned}
& \left|\left[S_{k}(x, y)-S_{k}\left(x, y^{\prime}\right)\right]-\left[S_{k}\left(x^{\prime}, y\right)-S_{k}\left(x^{\prime}, y^{\prime}\right)\right]\right| \\
& \quad \leq c\left(\frac{\rho\left(x, x^{\prime}\right)}{2^{-k}+\rho(x, y)}\right)^{\varepsilon^{\prime}}\left(\frac{\rho\left(y, y^{\prime}\right)}{2^{-k}+\rho(x, y)}\right)^{\varepsilon^{\prime}} \frac{2^{-k \sigma}}{\left(2^{-k}+\rho(x, y)\right)^{1+\sigma}}
\end{aligned}
$$

for $0<\varepsilon^{\prime}<\varepsilon, \sigma=\varepsilon-\varepsilon^{\prime}>0, \rho\left(x, x^{\prime}\right) \leq \frac{1}{2 A}\left(2^{-k}+\rho(x, y)\right)$ and $\rho\left(y, y^{\prime}\right) \leq$ $\frac{1}{2 A}\left(2^{-k}+\rho(x, y)\right)$

(v)

$$
\int_{X} S_{k}(x, y) d \mu(y)=\int_{X} S_{k}(x, y) d \mu(x)=1 \quad \text { for all } k \in \mathbb{Z} .
$$

See [DJS] for the existence of such a sequence of operators. All conditions are introduced and checked there except the condition (iv) in Definition 1.6. It is easy to see that the same construction in [DJS] also satisfies the condition (iv) in Definition 1.6. To define the Besov and Triebel-Lizorkin spaces on spaces of homogeneous type we also need the following definition (see [HS]).

Definition 1.7. Fix two exponents $0<\beta \leq \theta$ and $\gamma>0$. A function $f$ defined on $X$ is said to be a test function of type $(\beta, \gamma)$ centered at $x_{0} \in X$ with width $d>0$, 
if $f$ satisfies the following conditions:

$$
\begin{aligned}
& \text { (i) }|f(x)| \leq c \frac{d^{\gamma}}{\left(d+\rho\left(x, x_{0}\right)\right)^{1+\gamma}}, \\
& \begin{array}{l}
\text { (ii) }\left|f(x)-f\left(x^{\prime}\right)\right| \leq c\left[\frac{\rho\left(x, x^{\prime}\right)}{d+\rho\left(x, x_{0}\right)}\right]^{\beta} \frac{d^{\gamma}}{\left(d+\rho\left(x, x_{0}\right)\right)^{1+\gamma}} \\
\text { for } \rho\left(x, x^{\prime}\right) \leq \frac{1}{2 A}\left(d+\rho\left(x, x_{0}\right)\right), \\
\text { (iii) } \int_{X} f(x) d \mu(x)=0 .
\end{array}
\end{aligned}
$$

This definition was first introduced in $[\mathrm{M}]$ for the case $X=R^{n}$ with the condition (ii) in (1.8) replaced by

$$
\left|f(x)-f\left(x^{\prime}\right)\right| \leq c\left[\frac{\rho\left(x, x^{\prime}\right)}{d}\right]^{\beta}\left[\frac{d^{\gamma}}{\left(d+\rho\left(x, x_{0}\right)\right)^{1+\gamma}}+\frac{d^{\gamma}}{\left(d+\rho\left(x^{\prime}, x_{0}\right)\right)^{1+\gamma}}\right] .
$$

The collection of all test functions of type $(\beta, \gamma)$ centered at $x_{0} \in X$ with width $d>0$ will be denoted by $\mathcal{M}^{(\beta, \gamma)}\left(x_{0}, d\right)$. If $f \in \mathcal{M}^{(\beta, \gamma)}\left(x_{0}, d\right)$, the norm of $f$ in $\mathcal{M}^{(\beta, \gamma)}\left(x_{0}, d\right)$ is then defined by

$$
\|f\|_{\mathcal{M}^{(\beta, \gamma)}\left(x_{0}, d\right)}=\inf \{c \geq 0 \text { : (i) and (ii) of (1.8) hold }\} .
$$

Now fix a point $x_{0} \in X$ and denote the class of all $f \in \mathcal{M}^{(\beta, \gamma)}\left(x_{0}, 1\right)$ by $\mathcal{M}^{(\beta, \gamma)}$. It is easy to see that $\mathcal{M}^{(\beta, \gamma)}$ is a Banach space under the norm $\|f\|_{\mathcal{M}^{(\beta, \gamma)}}<\infty$. Just as the space of distributions $\mathcal{S}^{\prime}$ is defined on $R^{n}$, the dual space $\left(\mathcal{M}^{(\beta, \gamma)}\right)^{\prime}$ consists of all linear functionals $\ell$ from $\mathcal{M}^{(\beta, \gamma)}$ to $\mathbb{C}$ with the property that there exists a finite constant $c$ such that for all $f \in \mathcal{M}^{(\beta, \gamma)},|\ell(f)| \leq c\|f\|_{\mathcal{M}^{(\beta, \gamma)}}$. We denote the natural pairing of elements $h \in\left(\mathcal{M}^{(\beta, \gamma)}\right)^{\prime}$ and $f \in \mathcal{M}^{(\beta, \gamma)}$ by $\langle h, f\rangle$. It is also easy to see that $\mathcal{M}^{(\beta, \gamma)}\left(x_{1}, d\right)=\mathcal{M}^{(\beta, \gamma)}$ with equivalent norms for $x_{1} \in X$ and $d>0$. Thus, $\langle h, f\rangle$ is well defined for all $h \in\left(\mathcal{M}^{(\beta, \gamma)}\right)^{\prime}$ and all $f \in \mathcal{M}^{(\beta, \gamma)}\left(x_{1}, d\right)$ with $x_{1} \in X$ and $d>0$.

In [HS] the Besov and Triebel-Lizorkin spaces on homogeneous type were introduced by use of the family of operators $\left(D_{k}\right)_{k \in \mathbb{Z}}$ where $D_{k}=S_{k}-S_{k-1}$ and $\left(S_{k}\right)_{k \in \mathbb{Z}}$ is an approximation to the identity defined in Definition 1.6. More precisely, the Besov space $\dot{B}_{p}^{\alpha, q}$ for $-\varepsilon<\alpha<\varepsilon, 1 \leq p, q \leq \infty$, is the collection of all $f \in\left(\mathcal{M}^{(\beta, \gamma)}\right)^{\prime}$ with $0<\beta, \gamma<\varepsilon$ such that

$$
\|f\|_{\dot{B}_{p}^{\alpha, q}}=\left\{\sum_{k \in \mathbb{Z}}\left(2^{k \alpha}\left\|D_{k}(f)\right\|_{p}\right)^{q}\right\}^{1 / q}<\infty .
$$

The Triebel-Lizorkin space $\dot{F}_{p}^{\alpha, q}$ for $-\varepsilon<\alpha<\varepsilon, 1<p, q<\infty$, is the collection of all $f \in\left(\mathcal{M}^{(\beta, \gamma)}\right)^{\prime}$ with $0<\beta, \gamma<\varepsilon$ such that

$$
\|f\|_{\dot{F}_{p}^{\alpha, q}}=\left\|\left\{\sum_{k \in \mathbb{Z}}\left(2^{k \alpha}\left|D_{k}(f)\right|\right)^{q}\right\}^{1 / q}\right\|_{p}<\infty .
$$

In [HS] the above Besov and Triebel-Lizorkin spaces were proved to be independent of the choice of the approximations to the identity. The proofs were based on the continuous version of the Calderon type reproducing formula on spaces of homogeneous type obtained in [HS]. In [H1], using the continuous version of the Calderon 
formula in [HS], the Littlewood-Paley $S$-function and atomic decomposition, the above Triebel-Lizorkin spaces on spaces of homogeneous type were generalized to the case where $\max \left\{\frac{1}{1+\varepsilon}, \frac{1}{1+\alpha+\varepsilon}\right\}<p \leq 1 \leq q<\infty$. The method used in [H1], however, cannot be applied to either the Besov spaces for $p<1$ or the Triebel-Lizorkin spaces for $q<1$. In this paper, using the discrete Calderon reproducing formula developed in [H2], we obtain the Plancherel-Pôlya type inequalities. These inequalities give new characterizations of the Besov and Triebel-Lizorkin spaces introduced in [HS]. These new characterizations enable us to generalize the Besov spaces $\dot{B}_{p}^{\alpha, q}$ to the case where $-\varepsilon<\alpha<\varepsilon, \max \left(\frac{1}{1+\varepsilon}, \frac{1}{1+\varepsilon+\alpha}\right)<p \leq \infty$, and $0<q \leq \infty$, and the Triebel-Lizorkin spaces $\dot{F}_{p}^{\alpha, q}$ to the case where $-\varepsilon<\alpha<\varepsilon, \max \left\{\frac{1}{1+\varepsilon}, \frac{1}{1+\alpha+\varepsilon}\right\}<p$, $q<\infty$. Using the Plancherel-Pôlya type inequalities, we also prove that the $L^{p}$ norms of the Littlewood-Paley $G$-function and $S$-function are equivalent on spaces of homogeneous type. As a consequence, we obtain the Littlewood-Paley $G$-function characterization of the Hardy spaces on spaces of homogeneous type introduced in [MS2].

To state our results, we recall a result of Christ $([\mathrm{Ch}])$, which is an analogue of the Euclidean dyadic cubes.

Theorem 1.13. There exist a collection of open subsets $\left\{Q_{\tau}^{k} \subset X: k \in \mathbb{Z}, \tau \in I_{k}\right\}$, where $I_{k}$ denotes some (possibly finite) index set depending on $k$, and constants $\delta \in(0,1), a_{0}>0, \eta>0$ and $0<c_{1}, c_{2}<\infty$ such that

(i) $\mu\left(X \backslash \bigcup_{\tau} Q_{\tau}^{k}\right)=0$ for all $k \in \mathbb{Z}$,

(ii) If $\ell \geq k$, then either $Q_{\tau^{\prime}}^{\ell} \subset Q_{\tau}^{k}$ or $Q_{\tau^{\prime}}^{\ell} \cap Q_{\tau}^{k}=\varnothing$,

(iii) For each $(k, \tau)$ and each $\ell<k$, there is a unique $\tau^{\prime}$ such that $Q_{\tau}^{k} \subset Q_{\tau^{\prime}}^{\ell}$,

(iv) Diameter $\left(Q_{\tau}^{k}\right) \leq c_{1} \delta^{k}$,

(v) Each $Q_{\tau}^{k}$ contains some ball $B\left(z_{\tau}^{k}, a_{0} \delta^{k}\right)$.

We fix such a collection of open subsets and call all $Q_{\tau}^{k}$ in Theorem 1.13 the "dyadic cubes" in $X$. Without loss of generality, we may assume $\delta=\frac{1}{2}$ in Theorem 1.13. It is easy to check that our results and the proofs are independent of the choice of open subsets which satisfy the hypotheses of Theorem 1.13. Denote $Q_{\tau}^{k, \nu}$, $\nu=1,2, \ldots, N(k, \tau)$, the set of all cubes $Q_{\tau^{\prime}}^{k+j} \subseteq Q_{\tau}^{k}$ where $j$ is a positive fixed large integer. Denote by $y_{\tau}^{k, \nu}$ the points in $Q_{\tau}^{k, \nu}$.

Now the main results of this paper can be stated as follows.

Theorem 1 (The Plancherel-Pôlya type inequality). Suppose that $\left(S_{k}\right)_{k \in \mathbb{Z}}$ and $\left(P_{k}\right)_{k \in \mathbb{Z}}$ are approximations to the identity. Set $D_{k}=S_{k}-S_{k-1}$ and $Q_{k}=$ $P_{k}-P_{k-1}$. Then, for $f \in\left(\mathcal{M}^{(\beta, \gamma)}\right)^{\prime}$ with $0<\beta, \gamma<\varepsilon$, there exist two constants $c_{1}$ and $c_{2}$ such that if $-\varepsilon<\alpha<\varepsilon, \max \left(\frac{1}{1+\varepsilon}, \frac{1}{1+\varepsilon+\alpha}\right)<p \leq \infty$ and $0<q \leq \infty$,

$$
\begin{gathered}
\left\{\sum_{k \in \mathbb{Z}}\left(\sum_{\tau \in I_{k}} \sum_{\nu=1}^{N(k, \tau)}\left[\left(\mu\left(Q_{\tau}^{k, \nu}\right)\right)^{-\alpha+\frac{1}{p}} \sup _{z \in Q_{\tau}^{k, \nu}}\left|D_{k}(f)(z)\right|\right]^{p}\right)^{\frac{q}{p}}\right\}^{\frac{1}{q}} \\
\approx\left\{\sum_{k \in \mathbb{Z}}\left(\sum_{\tau \in I_{k}} \sum_{\nu=1}^{N(k, \tau)}\left[\left(\mu\left(Q_{\tau}^{k, \nu}\right)\right)^{-\alpha+\frac{1}{p}} \inf _{z \in Q_{\tau}^{k, \nu}}\left|Q_{k}(f)(z)\right|\right]^{p}\right)^{\frac{q}{p}}\right\}^{\frac{1}{q}} ;
\end{gathered}
$$


and if $-\varepsilon<\alpha<\varepsilon, \max \left(\frac{1}{1+\varepsilon}, \frac{1}{1+\varepsilon+\alpha}\right)<p, q<\infty$,

$$
\begin{gathered}
\left\|\left\{\sum_{k \in \mathbb{Z}} \sum_{\tau \in I_{k}} \sum_{\nu=1}^{N(k, \tau)}\left[\left(\mu\left(Q_{\tau}^{k, \nu}\right)\right)^{-\alpha} \sup _{z \in Q_{\tau}^{k, \nu}}\left|D_{k}(f)(z)\right| \chi_{Q_{\tau}^{k, \nu}}(x)\right]^{q}\right\}^{\frac{1}{q}}\right\|_{p} \\
\approx\left\|\left\{\sum_{k \in \mathbb{Z}} \sum_{\tau \in I_{k}} \sum_{\nu=1}^{N(k, \tau)}\left[\left(\mu\left(Q_{\tau}^{k, \nu}\right)\right)^{-\alpha} \inf _{z \in Q_{\tau}^{k, \nu}}\left|Q_{k}(f)(z)\right| \chi_{Q_{\tau}^{k, \nu}}(x)\right]^{q}\right\}^{\frac{1}{q}}\right\|_{p} .
\end{gathered}
$$

Theorem 2. Suppose that $\left(S_{k}\right)_{k \in \mathbb{Z}}$ is an approximation to the identity. Set $D_{k}=$ $S_{k}-S_{k-1}$. Then, for $f \in\left(\mathcal{M}^{(\beta, \gamma)}\right)^{\prime}$ with $0<\beta, \gamma<\varepsilon$,

$$
\begin{aligned}
& \left\{\sum_{k \in \mathbb{Z}}\left(\sum_{\tau \in I_{k}} \sum_{\nu=1}^{N(k, \tau)}\left[\left(\mu\left(Q_{\tau}^{k, \nu}\right)\right)^{-\alpha+\frac{1}{p}} \sup _{z \in Q_{\tau}^{k, \nu}}\left|D_{k}(f)(z)\right|\right]^{p}\right\}^{\frac{q}{p}}\right\}^{\frac{1}{q}} \\
& \approx\left\{\sum_{k \in \mathbb{Z}}\left(\sum_{\tau \in I_{k}} \sum_{\nu=1}^{N(k, \tau)}\left[\left(\mu\left(Q_{\tau}^{k, \nu}\right)\right)^{-\alpha+\frac{1}{p}} \inf _{z \in Q_{\tau}^{k, \nu}}\left|D_{k}(f)(z)\right|\right]^{p}\right)^{\frac{q}{p}}\right\}^{\frac{1}{q}} \\
& \approx\left\{\sum_{k \in \mathbb{Z}}\left(2^{k \alpha}\left\|D_{k}(f)\right\|_{p}\right)^{q}\right\}^{1 / q}
\end{aligned}
$$

where $-\varepsilon<\alpha<\varepsilon, \max \left(\frac{1}{1+\varepsilon}, \frac{1}{1+\varepsilon+\alpha}\right)<p \leq \infty$ and $0<q \leq \infty$, and

$$
\begin{aligned}
& \left\|\left\{\sum_{k \in \mathbb{Z}} \sum_{\tau \in I_{k}} \sum_{\nu=1}^{N(k, \tau)}\left[\left(\mu\left(Q_{\tau}^{k, \nu}\right)\right)^{-\alpha} \sup _{z \in Q_{\tau}^{k, \nu}}\left|D_{k}(f)(z)\right| \chi_{Q_{\tau}^{k, \nu}}(x)\right]^{q}\right\}^{\frac{1}{q}}\right\|_{p} \\
& \approx\left\|\left\{\sum_{k \in \mathbb{Z}} \sum_{\tau \in I_{k}} \sum_{\nu=1}^{N(k, \tau)}\left[\left(\mu\left(Q_{\tau}^{k, \nu}\right)\right)^{-\alpha} \inf _{z \in Q_{\tau}^{k, \nu}}\left|D_{k}(f)(z)\right| \chi_{Q_{\tau}^{k, \nu}}(x)\right]^{q}\right\}^{\frac{1}{q}}\right\|_{p} \\
& \approx\left\|\left\{\sum_{k \in \mathbb{Z}}\left(2^{k \alpha}\left|D_{k}(f)\right|\right)^{q}\right\}^{1 / q}\right\|
\end{aligned}
$$

where $-\varepsilon<\alpha<\varepsilon, \max \left\{\frac{1}{1+\varepsilon}, \frac{1}{1+\alpha+\varepsilon}\right\}<p, q<\infty$.

Theorem 2 gives new characterizations of the Besov and Triebel-Lizorkin spaces introduced in [HS] and allows us to generalize the Besov and Triebel-Lizorkin spaces on spaces of homogeneous type as follows.

Definition 1.18. Suppose that $-\varepsilon<\alpha<\varepsilon, \max \left(\frac{1}{1+\varepsilon}, \frac{1}{1+\varepsilon+\alpha}\right)<p \leq \infty$ and $0<q \leq \infty$. The Besov space $\dot{B}_{p}^{\alpha, q}$ is the collection of all $f \in\left(\mathcal{M}^{(\beta, \gamma)}\right)^{\prime}$ with $0<\beta$, $\gamma<\varepsilon$ such that

$$
\|f\|_{\dot{B}_{p}^{\alpha, q}}=\left\{\sum_{k \in \mathbb{Z}}\left(2^{k \alpha}\left\|D_{k}(f)\right\|_{p}\right)^{q}\right\}^{1 / q}<\infty .
$$


The Triebel-Lizorkin space $\dot{F}_{p}^{\alpha, q}$ for $-\varepsilon<\alpha<\varepsilon, \max \left\{\frac{1}{1+\varepsilon}, \frac{1}{1+\alpha+\varepsilon}\right\}<p, q<\infty$, is the collection of all $f \in\left(\mathcal{M}^{(\beta, \gamma)}\right)^{\prime}$ with $0<\beta, \gamma<\varepsilon$ such that

$$
\|f\|_{\dot{F}_{p}^{\alpha, q}}=\left\|\left\{\sum_{k \in \mathbb{Z}}\left(2^{k \alpha}\left|D_{k}(f)\right|\right)^{q}\right\}^{1 / q}\right\|_{p}<\infty .
$$

Theorem 3. Suppose that $\left(S_{k}\right)_{k \in \mathbb{Z}}$ is an approximation to the identity. Set $D_{k}=$ $S_{k}-S_{k-1}$. Then for $f \in\left(\mathcal{M}^{(\beta, \gamma)}\right)^{\prime}$ with $0<\beta, \gamma<\varepsilon$, there exist two constants $c_{1}$ and $c_{2}$ such that if $-\varepsilon<\alpha<\varepsilon, \max \left\{\frac{1}{1+\varepsilon}, \frac{1}{1+\alpha+\varepsilon}\right\}<p, q<\infty$,

$$
c_{1}\left\|S_{q}^{\alpha}(f)\right\|_{p} \leq\left\|\left\{\sum_{k \in \mathbb{Z}}\left(2^{k \alpha}\left|D_{k}(f)\right|\right)^{q}\right\}^{1 / q}\right\|_{p} \leq c_{2}\left\|S_{q}^{\alpha}(f)\right\|_{p}
$$

where the Littlewood-Paley S-function $S_{q}^{\alpha}(f)$ is defined by

$$
S_{q}^{\alpha}(f)(x)=\left\{\sum_{k \in \mathbb{Z}} \int_{\rho(x, y) \leq 2^{-k}} 2^{k}\left(2^{k \alpha}\left|D_{k}(f)(y)\right|\right)^{q} d \mu(y)\right\}^{1 / q} .
$$

As an immediate consequence of Theorem 3 and a result of [H1] we have

Theorem 4. $H^{p}(X)=\dot{F}_{p}^{0,2}(X)$ for $\frac{1}{1+\varepsilon}<p \leq 1$, where $H^{p}$ is the Hardy spaces on spaces of homogeneous type introduced by Macias and Segovia ([MS2]).

We prove Theorem 1 in section 2, and Theorems 2, 3 and 4 in section 3.

\section{Proof of Theorem 1}

To prove Theorem 1, we need the following discrete Calderon type reproducing formula on spaces of homogeneous type obtained in [H2].

Theorem $2.1([\mathrm{H} 2])$. Suppose that $\left(S_{k}\right)_{k \in \mathbb{Z}}$ is an approximation to the identity defined in Definition 1.6. Set $D_{k}=S_{k}-S_{k-1}$. Then there exists a family of functions $\left(\widetilde{D}_{k}(x, y)\right)_{k \in \mathbb{Z}}$ such that for all fixed $y_{\tau}^{k, \nu} \in Q_{\tau}^{k, \nu}$ and all $f \in\left(\mathcal{M}^{(\beta, \gamma)}\right)^{\prime}$ with $0<\beta, \gamma<\varepsilon$,

$$
f(x)=\sum_{k \in \mathbb{Z}} \sum_{\tau \in I_{k}} \sum_{\nu=1}^{N(k, \tau)} \mu\left(Q_{\tau}^{k, \nu}\right) \widetilde{D}_{k}\left(x, y_{\tau}^{k, \nu}\right) D_{k}(f)\left(y_{\tau}^{k, \nu}\right)
$$

where the series converges in $\left(\mathcal{M}^{\left(\beta^{\prime}, \gamma^{\prime}\right)}\right)^{\prime}$ with $\beta^{\prime}>\beta$ and $\gamma^{\prime}>\gamma$. Moreover, $\widetilde{D}_{k}(x, y), k \in \mathbb{Z}$, satisfy the following estimates: for $0<\varepsilon^{\prime}<\varepsilon$, where $\varepsilon$ is the regularity exponent of $S_{k}$, there exists a constant $c>0$ which depends only on $\varepsilon$ and $\varepsilon^{\prime}$, such that

$$
\begin{gathered}
\left|\widetilde{D}_{k}(x, y)\right| \leq c \frac{2^{-k \varepsilon^{\prime}}}{\left(2^{-k}+\rho(x, y)\right)^{1+\varepsilon^{\prime}}}, \\
\left|\widetilde{D}_{k}(x, y)-\widetilde{D}_{k}\left(x^{\prime}, y\right)\right| \leq c\left(\frac{\rho\left(x, x^{\prime}\right)}{2^{-k}+\rho(x, y)}\right)^{\varepsilon^{\prime}} \frac{2^{-k \varepsilon^{\prime}}}{\left(2^{-k}+\rho(x, y)\right)^{1+\varepsilon^{\prime}}} \\
\quad \text { for } \rho\left(x, x^{\prime}\right) \leq \frac{1}{2 A}\left[2^{-k}+\rho(x, y)\right],
\end{gathered}
$$




$$
\int \widetilde{D}_{k}(x, y) d \mu(x)=\int \widetilde{D}_{k}(x, y) d \mu(y)=0 \quad \text { for all } k \in \mathbb{Z} .
$$

We now prove (1.14) of Theorem 1. By Theorem 2.1, for any $z \in Q_{\tau}^{k, \nu}$ we have

$$
D_{k}(f)(z)=\sum_{k^{\prime} \in \mathbb{Z}} \sum_{\tau^{\prime} \in I_{k^{\prime}}} \sum_{\nu^{\prime}=1}^{N\left(k^{\prime}, \tau^{\prime}\right)} \mu\left(Q_{\tau^{\prime}}^{k^{\prime}, \nu^{\prime}}\right)\left(D_{k} \widetilde{Q}_{k}\right)\left(z, y_{\tau^{\prime}}^{k^{\prime}, \nu^{\prime}}\right) Q_{k^{\prime}}(f)\left(y_{\tau^{\prime}}^{k^{\prime}, \nu^{\prime}}\right)
$$

where $y_{\tau^{\prime}}^{k^{\prime}, \nu^{\prime}}$ are any fixed points in $Q_{\tau^{\prime}}^{k^{\prime}, \nu^{\prime}}$.

Choose $\varepsilon^{\prime \prime}<\varepsilon^{\prime}<\varepsilon$ so that $|\alpha|<\varepsilon^{\prime \prime}$ and $p>\max \left\{\frac{1}{1+\varepsilon^{\prime \prime}}, \frac{1}{1+\alpha+\varepsilon^{\prime \prime}}\right\}$. By the estimate (2.17) on page 66 in [H1], there exists a constant $c$ such that

$$
\left|\left(D_{k} \widetilde{Q}_{k}\right)\left(z, y_{\tau^{\prime}}^{k^{\prime}, \nu^{\prime}}\right)\right| \leq c 2^{-\left|k-k^{\prime}\right| \varepsilon^{\prime \prime}} \mu \frac{2^{-\left(k \wedge k^{\prime}\right) \varepsilon^{\prime}}}{\left(2^{-\left(k \wedge k^{\prime}\right)}+\rho\left(z, y_{\tau^{\prime}}^{k^{\prime}, \nu^{\prime}}\right)\right)^{1+\varepsilon^{\prime}}}
$$

where $a \wedge b$ denotes the minimum of $a$ and $b$. Thus,

$$
\begin{aligned}
\sup _{z \in Q_{\tau}^{k, \nu}}\left|D_{k}(f)(z)\right| \leq c \sum_{k^{\prime} \in \mathbb{Z}} \sum_{\tau^{\prime} \in I_{k^{\prime}}} \sum_{\nu^{\prime}=1}^{N\left(k^{\prime}, \tau^{\prime}\right)} 2^{-\left|k-k^{\prime}\right| \varepsilon^{\prime \prime}} \mu\left(Q_{\tau^{\prime}}^{k^{\prime}, \nu^{\prime}}\right) \\
\cdot \frac{2^{-\left(k \wedge k^{\prime}\right) \varepsilon^{\prime}}}{\left(2^{-\left(k \wedge k^{\prime}\right)}+\rho\left(z_{\tau}^{k, \nu}, y_{\tau^{\prime}}^{k^{\prime}, \nu^{\prime}}\right)\right)^{1+\varepsilon^{\prime}}}\left|Q_{k^{\prime}}(f)\left(y_{\tau^{\prime}}^{k^{\prime}, \nu^{\prime}}\right)\right|
\end{aligned}
$$

where $z_{\tau}^{k, \nu}$ are any fixed points in $Q_{\tau}^{k, \nu}$.

Now, by Holder for $p>1$ and $(a+b)^{p} \leq a^{p}+b^{p}$ for $p \leq 1$, and the facts that if $p>\frac{1}{1+\varepsilon^{\prime}}$,

$$
\begin{aligned}
& \sum_{\tau^{\prime} \in I_{k^{\prime}}} \sum_{\nu^{\prime}=1}^{N\left(k^{\prime}, \tau^{\prime}\right)} \mu\left(Q_{\tau^{\prime}}^{k^{\prime}, \nu^{\prime}}\right)\left[\frac{2^{-\left(k \wedge k^{\prime}\right) \varepsilon^{\prime}}}{\left(2^{-\left(k \wedge k^{\prime}\right)}+\rho\left(z_{\tau}^{k, \nu}, y_{\tau^{\prime}}^{k^{\prime}, \nu^{\prime}}\right)\right)^{1+\varepsilon^{\prime}}}\right]^{(p \wedge 1)} \\
& \quad \leq c \int_{X}\left[\frac{2^{-\left(k \wedge k^{\prime}\right) \varepsilon^{\prime}}}{\left(2^{-\left(k \wedge k^{\prime}\right)}+\rho\left(z_{\tau}^{k, \nu}, y\right)\right)^{1+\varepsilon^{\prime}}}\right]^{(p \wedge 1)} d \mu(y) \\
& \quad \leq c 2^{-\left(k \wedge k^{\prime}\right)[1-(p \wedge 1)]}
\end{aligned}
$$

and

$$
\sup _{k} \sum_{k^{\prime}} 2^{\left(k-k^{\prime}\right) \alpha_{2}-\left|k-k^{\prime}\right| \varepsilon^{\prime \prime}}<\infty
$$


we obtain

$$
\begin{aligned}
& \sum_{\tau \in I_{k}} \sum_{\nu=1}^{N(k, \tau)}\left[\left(\mu\left(Q_{\tau}^{k, \nu}\right)\right)^{-\alpha+\frac{1}{p}} \sup _{z \in Q_{\tau}^{k, \nu}}\left|D_{k}(f)(z)\right|\right]^{p} \\
& \leq c \sum_{\tau \in I_{k}} \sum_{\nu=1}^{N(k, \tau)}\left[\sum_{k^{\prime}} \sum_{\tau^{\prime} \in I_{k^{\prime}}} \sum_{\nu^{\prime}=1}^{N\left(k^{\prime}, \tau^{\prime}\right)} 2^{\left(k-k^{\prime}\right) \alpha_{2}-\left|k-k^{\prime}\right| \varepsilon^{\prime \prime}} 2^{-k / p} 2^{-k^{\prime}}\right. \\
& \left.\quad \times \frac{2^{-\left(k \wedge k^{\prime}\right) \varepsilon^{\prime}}}{\left(2^{-\left(k \wedge k^{\prime}\right)}+\rho\left(z_{\tau}^{k, \nu}, y_{\tau^{\prime}}^{k^{\prime}, \nu^{\prime}}\right)\right)^{1+\varepsilon^{\prime}}} 2^{k^{\prime} \alpha}\left|Q_{k^{\prime}}(f)\left(y_{\tau^{\prime}}^{k^{\prime}, \nu^{\prime}}\right)\right|\right]^{p} \\
& \leq c \sum_{k^{\prime}} \sum_{\tau^{\prime} \in I_{k^{\prime}}} \sum_{\nu^{\prime}=1}^{N\left(k^{\prime}, \tau^{\prime}\right)} 2^{\left(k-k^{\prime}\right) \alpha(p \wedge 1)} 2^{-\left|k-k^{\prime}\right| \varepsilon^{\prime \prime}(p \wedge 1)} \\
& \times 2^{-\left(k \wedge k^{\prime}\right)[1-(p \wedge 1)]} 2^{-k^{\prime}(p \wedge 1)}\left[2^{k^{\prime} \alpha}\left|Q_{k^{\prime}}(f)\left(y_{\tau^{\prime}}^{k^{\prime}, \nu^{\prime}}\right)\right|\right]^{p} .
\end{aligned}
$$

Thus, by Holder for $q / p>1$ and $(a+b)^{q / p} \leq a^{q / p}+b^{q / p}$ for $q / p \leq 1$, we have

$$
\begin{aligned}
& \left\{\sum_{k \in \mathbb{Z}}\left(\sum_{\tau \in I_{k}} \sum_{\nu=1}^{N(k, \tau)}\left[\left(\mu\left(Q_{\tau}^{k, \nu}\right)\right)^{-\alpha+\frac{1}{p}} \sup _{z \in Q_{\tau}^{k, \nu}}\left|D_{k}(f)(z)\right|\right]^{p}\right)^{\frac{q}{p}}\right\}^{\frac{1}{q}} \\
& \leq c\left\{\sum _ { k \in \mathbb { Z } } \left(\sum_{k^{\prime} \in \mathbb{Z}} 2^{\left(k-k^{\prime}\right) \alpha(p \wedge 1)} 2^{-\left|k-k^{\prime}\right| \varepsilon^{\prime \prime}(p \wedge 1)} 2^{-\left(k \wedge k^{\prime}\right)[1-(p \wedge 1)]} 2^{-k^{\prime}[(p \wedge 1)-1]}\right.\right. \\
& \left.\left.\times \sum_{\tau^{\prime} \in I_{k^{\prime}}} \sum_{\nu^{\prime}=1}^{N\left(k^{\prime}, \tau^{\prime}\right)}\left[\left(\mu\left(Q_{\tau^{\prime}}^{k^{\prime}, \nu^{\prime}}\right)\right)^{-\alpha+\frac{1}{p}}\left|Q_{k^{\prime}}(f)\left(y_{\tau^{\prime}}^{k^{\prime}, \nu^{\prime}}\right)\right|\right]^{p}\right)^{\frac{q}{p}}\right\}^{\frac{1}{q}} \\
& \leq c\left\{\sum_{k \in \mathbb{Z}} \sum_{k^{\prime} \in \mathbb{Z}}\left(2^{\left(k-k^{\prime}\right) \alpha(p \wedge 1)} 2^{-\left|k-k^{\prime}\right| \varepsilon^{\prime \prime}(p \wedge 1)} 2^{-\left(k \wedge k^{\prime}\right)[1-(p \wedge 1)]} 2^{-k^{\prime}[(p \wedge 1)-1]}\right)^{\left(\frac{q}{p} \wedge 1\right)}\right. \\
& \left.\times\left(\sum_{\tau^{\prime} \in I_{k^{\prime}}} \sum_{\nu^{\prime}=1}^{N\left(k^{\prime}, \tau^{\prime}\right)}\left[\left(\mu\left(Q_{\tau^{\prime}}^{k^{\prime}, \nu^{\prime}}\right)\right)^{-\alpha+\frac{1}{p}}\left|Q_{k^{\prime}}(f)\left(y_{\tau^{\prime}}^{k^{\prime}, \nu^{\prime}}\right)\right|\right]^{p}\right)^{\frac{q}{p}}\right\}^{\frac{1}{q}} \\
& \leq c\left\{\sum_{k^{\prime} \in \mathbb{Z}}\left(\sum_{\tau^{\prime} \in I_{k^{\prime}}} \sum_{\nu^{\prime}=1}^{N\left(k^{\prime}, \tau^{\prime}\right)}\left[\left(\mu\left(Q_{\tau^{\prime}}^{k^{\prime}, \nu^{\prime}}\right)\right)^{-\alpha+\frac{1}{p}}\left|Q_{k^{\prime}}(f)\left(y_{\tau^{\prime}}^{k^{\prime}, \nu^{\prime}}\right)\right|\right]^{p}\right)^{\frac{q}{p}}\right\}^{\frac{1}{q}}
\end{aligned}
$$

since if $|\alpha|<\varepsilon^{\prime \prime}$ and $p>\frac{1}{1+\alpha+\varepsilon^{\prime \prime}}$ we have

$$
\sup _{k} \sum_{k^{\prime} \in \mathbb{Z}} 2^{\left(k-k^{\prime}\right) \alpha(p \wedge 1)} 2^{-\left|k-k^{\prime}\right| \varepsilon^{\prime \prime}(p \wedge 1)} 2^{-\left(k \wedge k^{\prime}\right)[1-(p \wedge 1)]} 2^{-k^{\prime}[(p \wedge 1)-1]}<\infty
$$

and

$$
\sup _{k^{\prime}} \sum_{k^{\prime} \in \mathbb{Z}}\left\{2^{\left(k-k^{\prime}\right) \alpha(p \wedge 1)} 2^{-\left|k-k^{\prime}\right| \varepsilon^{\prime \prime}(p \wedge 1)} 2^{-\left(k \wedge k^{\prime}\right)[1-(p \wedge 1)]} 2^{-k^{\prime}[(p \wedge 1)-1]}\right\}^{\left(\frac{q}{p} \wedge 1\right)}<\infty .
$$


Since (2.7) holds for all $y_{\tau^{\prime}}^{k^{\prime}, \nu^{\prime}} \in Q_{\tau^{\prime}}^{k^{\prime}, \nu^{\prime}}$, it is easy to see that (2.7) implies (1.14). We now return to the proof of (1.15). As above, we have

$$
\begin{gathered}
\left\{\sum_{k \in \mathbb{Z}} \sum_{\tau \in I_{k}} \sum_{\nu=1}^{N(k, \tau)}\left[\left(\mu\left(Q_{\tau}^{k, \nu}\right)\right)^{-\alpha} \sup _{z \in Q_{\tau}^{k, \nu}}\left|D_{k}(f)(z)\right| \chi_{Q_{\tau}^{k, \nu}}(x)\right]^{q}\right\}^{\frac{1}{q}} \\
\leq c\left\{\sum _ { k \in \mathbb { Z } } 2 ^ { \alpha k q } \left[\sum_{k^{\prime}} \sum_{\tau^{\prime} \in I_{k^{\prime}}} \sum_{\nu^{\prime}=1}^{N\left(k^{\prime}, \tau^{\prime}\right)} 2^{-\left|k-k^{\prime}\right| \varepsilon^{\prime \prime}} 2^{-k^{\prime}}\right.\right. \\
\left.\left.\times \frac{2^{-\left(k \wedge k^{\prime}\right) \varepsilon^{\prime}}}{\left(2^{-\left(k \wedge k^{\prime}\right)}+\rho\left(x, y_{\tau^{\prime}}^{k^{\prime}, \nu^{\prime}}\right)\right)^{1+\varepsilon^{\prime}}}\left|Q_{k^{\prime}}(f)\left(y_{\tau^{\prime}}^{k^{\prime}, \nu^{\prime}}\right)\right|\right]^{q}\right\}^{\frac{1}{q}}
\end{gathered}
$$

By an estimate in [FJ], pp. 147-148,

$$
\begin{aligned}
& \sum_{\tau^{\prime} \in I_{k^{\prime}}} \sum_{\nu^{\prime}=1}^{N\left(k^{\prime}, \tau^{\prime}\right)} \frac{2^{-\left(k \wedge k^{\prime}\right) \varepsilon^{\prime}}}{\left(2^{-\left(k \wedge k^{\prime}\right)}+\rho\left(x, y_{\tau^{\prime}}^{k^{\prime}, \nu^{\prime}}\right)\right)^{1+\varepsilon^{\prime}}}\left|Q_{k^{\prime}}(f)\left(y_{\tau^{\prime}}^{k^{\prime}, \nu^{\prime}}\right)\right| \\
& \quad \leq c 2^{\left(k \wedge k^{\prime}\right)} 2^{\left[k^{\prime}-\left(k \wedge k^{\prime}\right)\right] / r}\left\{M\left(\sum_{\tau^{\prime} \in I_{k^{\prime}}} \sum_{\nu^{\prime}=1}^{N\left(k^{\prime}, \tau^{\prime}\right)}\left|Q_{k^{\prime}}(f)\left(y_{\tau^{\prime}}^{k^{\prime}, \nu^{\prime}}\right)\right| \chi_{Q_{\tau^{\prime}}^{k^{\prime}, \nu^{\prime}}}\right)^{r}\right\}^{\frac{1}{r}}
\end{aligned}
$$

where $M$ is the Hardy-Littlewood maximal function and $r>\frac{1}{1+\varepsilon^{\prime \prime}}$, we obtain, by Holder for $q>1$ and $(a+b)^{q} \leq a^{q}+b^{q}$ for $q \leq 1$,

$$
\begin{aligned}
& \left\{\sum_{k \in \mathbb{Z}} \sum_{\tau \in I_{k}} \sum_{\nu=1}^{N(k, \tau)}\left[\left(\mu\left(Q_{\tau}^{k, \nu}\right)\right)^{-\alpha} \sup _{z \in Q_{\tau}^{k, \nu}}\left|D_{k}(f)(z)\right| \chi_{Q_{\tau}^{k, \nu}}(x)\right]^{q}\right\}^{\frac{1}{q}} \\
& \leq c\left\{\sum _ { k \in \mathbb { Z } } \left[\sum_{k^{\prime} \in \mathbb{Z}} 2^{\left(k-k^{\prime}\right) \alpha} 2^{-\left|k-k^{\prime}\right| \varepsilon^{\prime \prime}} 2^{\left(k \wedge k^{\prime}\right)} 2^{-k^{\prime}} 2^{\left[k^{\prime}-\left(k \wedge k^{\prime}\right)\right] / r}\right.\right. \\
& \left.\left.\times\left(M\left(\sum_{\tau^{\prime} \in I_{k^{\prime}}} \sum_{\nu^{\prime}=1}^{N\left(k^{\prime}, \tau^{\prime}\right)}\left(\mu\left(Q_{\tau^{\prime}}^{k^{\prime}, \nu^{\prime}}\right)\right)^{-\alpha}\left|Q_{k^{\prime}}(f)\left(y_{\tau^{\prime}}^{k^{\prime}, \nu^{\prime}}\right)\right| \chi_{Q_{\tau^{\prime}}^{k^{\prime}, \nu^{\prime}}}\right)^{r}\right)^{\frac{1}{r}}(x)\right]^{q}\right\}^{\frac{1}{q}} \\
& \leq c\left\{\sum_{k \in \mathbb{Z}} \sum_{k^{\prime} \in \mathbb{Z}}\left(2^{\left(k-k^{\prime}\right) \alpha} 2^{-\left|k-k^{\prime}\right| \varepsilon^{\prime \prime}} 2^{\left(k \wedge k^{\prime}\right)} 2^{-k^{\prime}} 2^{\left[k^{\prime}-\left(k \wedge k^{\prime}\right)\right] / r}\right)^{(q \wedge 1)}\right. \\
& \left.\times\left(M\left(\sum_{\tau^{\prime} \in I_{k^{\prime}}} \sum_{\nu^{\prime}=1}^{N\left(k^{\prime}, \tau^{\prime}\right)}\left(\mu\left(Q_{\tau^{\prime}}^{k^{\prime}, \nu^{\prime}}\right)\right)^{-\alpha}\left|Q_{k^{\prime}}(f)\left(y_{\tau^{\prime}}^{k^{\prime}, \nu^{\prime}}\right)\right| \chi_{Q_{\tau^{\prime}}^{k^{\prime}, \nu^{\prime}}}\right)^{r}\right)^{\frac{q}{r}}(x)\right\}^{\frac{1}{q}} \\
& \leq c\left\{\sum_{k^{\prime} \in \mathbb{Z}}\left[\left(M\left(\sum_{\tau^{\prime} \in I_{k^{\prime}}} \sum_{\nu^{\prime}=1}^{N\left(k^{\prime}, \tau^{\prime}\right)}\left(\mu\left(Q_{\tau^{\prime}}^{k^{\prime}, \nu^{\prime}}\right)\right)^{-\alpha}\left|Q_{k^{\prime}}(f)\left(y_{\tau^{\prime}}^{k^{\prime}, \nu^{\prime}}\right)\right| \chi_{Q_{\tau^{\prime}}^{k^{\prime}, \nu^{\prime}}}\right)^{r}\right)^{\frac{1}{r}}(x)\right]^{q}\right\}^{\frac{1}{q}}
\end{aligned}
$$


since if $|\alpha|<\varepsilon^{\prime \prime}$ and $p, q>\max \left\{\frac{1}{1+\varepsilon^{\prime \prime}}, \frac{1}{1+\alpha+\varepsilon^{\prime \prime}}\right\}$, we can choose $r$ so that $r>$ $\max \left(\frac{1}{1+\varepsilon^{\prime \prime}}, \frac{1}{1+\alpha+\varepsilon^{\prime \prime}}\right), p>r$ and $q>r$, such that

$$
\sup _{k} \sum_{k^{\prime} \in \mathbb{Z}} 2^{\left(k-k^{\prime}\right) \alpha} 2^{-\left|k-k^{\prime}\right| \varepsilon^{\prime \prime}} 2^{\left(k \wedge k^{\prime}\right)} 2^{-k^{\prime}} 2^{\left[k^{\prime}-\left(k \wedge k^{\prime}\right)\right] / r}<\infty
$$

and

$$
\sup _{k^{\prime}} \sum_{k \in \mathbb{Z}}\left(2^{\left(k-k^{\prime}\right) \alpha} 2^{-\left|k-k^{\prime}\right| \varepsilon^{\prime \prime}} 2^{\left(k \wedge k^{\prime}\right)} 2^{-k^{\prime}} 2^{\left[k^{\prime}-\left(k \wedge k^{\prime}\right)\right] / r}\right)^{(q \wedge 1)}<\infty .
$$

Since (2.8) holds for all $y_{\tau^{\prime}}^{k^{\prime}, \nu^{\prime}} \in Q_{\tau^{\prime}}^{k^{\prime}, \nu^{\prime}}$, we have

$$
\begin{aligned}
& \left\{\sum_{k \in \mathbb{Z}} \sum_{\tau \in I_{k}} \sum_{\nu=1}^{N(k, \tau)}\left[\left(\mu\left(Q_{\tau}^{k, \nu}\right)\right)^{-\alpha} \sup _{z \in Q_{\tau}^{k, \nu}}\left|D_{k}(f)(z)\right| \chi_{Q_{\tau}^{k, \nu}}(x)\right]^{q}\right\}^{\frac{1}{q}} \\
& \leq c\left\{\sum_{k^{\prime} \in \mathbb{Z}}\left[\left(M\left(\sum_{\tau^{\prime} \in I_{k^{\prime}}} \sum_{\nu^{\prime}=1}^{N\left(k^{\prime}, \tau^{\prime}\right)}\left[\left(\mu\left(Q_{\tau^{\prime}}^{k^{\prime}, \nu^{\prime}}\right)\right)^{-\alpha} \inf _{z \in Q_{\tau^{\prime}}^{k^{\prime}, \nu^{\prime}}} \mid Q_{k^{\prime}}(f)(z) \chi_{Q_{\tau^{\prime}}^{k^{\prime}, \nu^{\prime}}}\right]\right)^{r}\right)^{\frac{1}{r}}(x)\right]^{q}\right\}^{\frac{1}{q}} .
\end{aligned}
$$

Now, by the Fefferman-Stein vector-valued maximal function inequality ([FS]), we obtain

$$
\begin{gathered}
\left\|\left\{\sum_{k \in \mathbb{Z}} \sum_{\tau \in I_{k}} \sum_{\nu=1}^{N(k, \tau)}\left[\left(\mu\left(Q_{\tau}^{k, \nu}\right)\right)^{-\alpha} \sup _{z \in Q_{\tau}^{k, \nu}}\left|D_{k}(f)(z)\right| \chi_{Q_{\tau}^{k, \nu}}(x)\right]^{q}\right\}^{\frac{1}{q}}\right\|_{p} \\
\leq c\left\|\left\{\sum_{k^{\prime} \in \mathbb{Z}} \sum_{\tau^{\prime} \in I_{k^{\prime}}} \sum_{\nu^{\prime}=1}^{N\left(k^{\prime}, \tau^{\prime}\right)}\left[\left(\mu\left(Q_{\tau^{\prime}}^{k^{\prime}, \nu^{\prime}}\right)\right)^{-\alpha} \inf _{z \in Q_{\tau^{\prime}}^{k^{\prime}, \nu^{\prime}}}\left|Q_{k^{\prime}}(f)(z)\right| \chi_{Q_{\tau^{\prime}}^{k^{\prime}, \nu^{\prime}}}\right]^{q}\right\}^{\frac{1}{q}}\right\|_{p} .
\end{gathered}
$$

\section{Proofs of Theorems 2,3 And 4}

We now prove Theorem 2. We first have

$$
\begin{aligned}
& \left\{\sum_{k \in \mathbb{Z}}\left(\sum_{\tau \in I_{k}} \sum_{\nu=1}^{N(k, \tau)}\left[\left(\mu\left(Q_{\tau}^{k, \nu}\right)\right)^{-\alpha+\frac{1}{p}} \inf _{z \in Q_{\tau}^{k, \nu}}\left|D_{k}(f)(z)\right|\right]^{p}\right)^{\frac{q}{p}}\right\}^{\frac{1}{p}} \\
& \leq c\left\{\sum_{k \in \mathbb{Z}}\left(\sum_{\tau \in I_{k}} \sum_{\nu=1}^{N(k, \tau)} 2^{\alpha k p} \int_{Q_{\tau}^{k, \nu}}\left|D_{k}(f)(z)\right|^{p}\right)^{\frac{q}{p}}\right\}^{\frac{1}{q}} \\
& =c\left\{\sum_{k \in \mathbb{Z}}\left(2^{\alpha k p} \int_{X}\left|D_{k}(f)(z)\right|^{p}\right)^{\frac{q}{p}}\right\}^{\frac{1}{q}}=c\left\{\sum_{k \in \mathbb{Z}}\left(2^{\alpha k}\left\|D_{k}(f)(z)\right\|_{p}\right)^{q}\right\}^{\frac{1}{q}} .
\end{aligned}
$$


We also have,

$$
\begin{gathered}
\left\{\sum_{k \in \mathbb{Z}}\left(\sum_{\tau \in I_{k}} \sum_{\nu=1}^{N(k, \tau)}\left[\left(\mu\left(Q_{\tau}^{k, \nu}\right)\right)^{-\alpha+\frac{1}{p}} \sup _{z \in Q_{\tau}^{k, \nu}}\left|D_{k}(f)(z)\right|\right]^{p}\right)^{\frac{q}{p}}\right\}^{\frac{1}{q}} \\
\geq c\left\{\sum_{k \in \mathbb{Z}}\left(\sum_{\tau \in I_{k}} \sum_{\nu=1}^{N(k, \tau)} 2^{\alpha k p} \int_{Q_{\tau}^{k, \nu}}\left|D_{k}(f)(z)\right|^{p}\right)^{\frac{q}{p}}\right\}^{\frac{1}{q}} \\
=c\left\{\sum_{k \in \mathbb{Z}}\left(2^{\alpha k p} \int_{X}\left|D_{k}(f)(z)\right|^{p}\right)^{\frac{q}{p}}\right\}^{\frac{1}{q}}=c\left\{\sum_{k \in \mathbb{Z}}\left(2^{\alpha k}\left\|D_{k}(f)(z)\right\|_{p}\right)^{q}\right\}^{\frac{1}{q}} .
\end{gathered}
$$

Similarly,

$$
\begin{gathered}
\left\|\left\{\sum_{k \in \mathbb{Z}} \sum_{\tau \in I_{k}} \sum_{\nu=1}^{N(k, \tau)}\left[\left(\mu\left(Q_{\tau}^{k, \nu}\right)\right)^{-\alpha} \inf _{z \in Q_{\tau}^{k, \nu}}\left|Q_{k}(f)(z)\right| \chi_{Q_{\tau}^{k, \nu}}\right]^{q}\right\}^{\frac{1}{q}}\right\|_{p} \\
\leq c\left\|\left\{\sum_{k \in \mathbb{Z}} 2^{\alpha k q}\left|Q_{k}(f)(x)\right|^{q}\right\}^{\frac{1}{q}}\right\|_{p}
\end{gathered}
$$

and

$$
\begin{gathered}
\left\|\left\{\sum_{k \in \mathbb{Z}} \sum_{\tau \in I_{k}} \sum_{\nu=1}^{N(k, \tau)}\left[\left(\mu\left(Q_{\tau}^{k, \nu}\right)\right)^{-\alpha} \sup _{z \in Q_{\tau}^{k, \nu}}\left|Q_{k}(f)(z)\right| \chi_{Q_{\tau}^{k, \nu}}\right]^{q}\right\}^{\frac{1}{q}}\right\|_{p} \\
\geq c\left\|\left\{\sum_{k \in \mathbb{Z}} 2^{\alpha k q}\left|Q_{k}(f)(x)\right|^{q}\right\}^{\frac{1}{q}}\right\|_{p} .
\end{gathered}
$$

Now the proof of Theorem 2 follows from (3.1), (3.2), (3.3), (3.4) and Theorem 1.

To see Theorem 3, note that if $c$ is a fixed constant, then by (2.6) we obtain

$$
\begin{aligned}
\sup _{z \in c Q_{\tau}^{k, \nu}}\left|D_{k}(f)(z)\right| \leq c \sum_{k^{\prime} \in \mathbb{Z}} \sum_{\tau^{\prime} \in I_{k^{\prime}}} & \sum_{\nu^{\prime}=1}^{N\left(k^{\prime}, \tau^{\prime}\right)} 2^{-\left|k-k^{\prime}\right| \varepsilon^{\prime}} \mu\left(Q_{\tau^{\prime}}^{k^{\prime}, \nu^{\prime}}\right) \\
& \times \frac{2^{-\left(k \wedge k^{\prime}\right) \varepsilon^{\prime}}}{\left(2^{-\left(k \wedge k^{\prime}\right)}+\rho\left(z_{\tau}^{k, \nu}, y_{\tau^{\prime}}^{k^{\prime}, \nu^{\prime}}\right)\right)^{1+\varepsilon^{\prime}}} Q_{k^{\prime}}(f)\left(y_{\tau^{\prime}}^{k^{\prime}, \nu^{\prime}}\right)
\end{aligned}
$$

where $z_{\tau}^{k, \nu}$ are any fixed points in $Q_{\tau}^{k, \nu}$. 
Thus, by the same proof, we have

$$
\begin{aligned}
& \left\|\left\{\sum_{k \in \mathbb{Z}} \sum_{\tau \in I_{k}} \sum_{\nu=1}^{N(k, \tau)}\left[\left(\mu\left(Q_{\tau}^{k, \nu}\right)\right)^{-\alpha} \sup _{z \in c Q_{\tau}^{k, \nu}}\left|D_{k}(f)(z)\right| \chi_{Q_{\tau}^{k, \nu}}(x)\right]^{q}\right\}^{\frac{1}{q}}\right\|_{p} \\
& \approx\left\|\left\{\sum_{k \in \mathbb{Z}} \sum_{\tau \in I_{k}} \sum_{\nu=1}^{N(k, \tau)}\left[\left(\mu\left(Q_{\tau}^{k, \nu}\right)\right)^{-\alpha} \inf _{z \in Q_{\tau}^{k, \nu}}\left|D_{k}(f)(z)\right| \chi_{Q_{\tau}^{k, \nu}}(x)\right]^{q}\right\}^{\frac{1}{q}}\right\|_{p} \\
& \approx\left\|\left\{\sum_{k \in \mathbb{Z}}\left(2^{k \alpha}\left|D_{k}(f)\right|\right)^{q}\right\}^{1 / q}\right\|_{p}
\end{aligned}
$$

where $c$ is any fixed constant.

For some fixed constant $c$ we have

$$
\begin{aligned}
& \left\|\left\{\sum_{k \in \mathbb{Z}} \int_{\rho(x, y) \leq 2^{-k}} 2^{k}\left(2^{k \alpha}\left|D_{k}(f)(y)\right|\right)^{q} d \mu(y)\right\}^{1 / q}\right\|_{p} \\
& =\left\|\left\{\sum_{k \in \mathbb{Z}} \sum_{\tau \in I_{k}} \sum_{\nu=1}^{N(k, \tau)} \int_{\rho(x, y) \leq 2^{-k}} 2^{k}\left(2^{k \alpha}\left|D_{k}(f)(y)\right|\right)^{q} \chi_{Q_{\tau}^{k, \nu}}(x) d \mu(y)\right\}^{1 / q}\right\|_{p} \\
& \leq\left\|\left\{\sum_{k \in \mathbb{Z}} \sum_{\tau \in I_{k}} \sum_{\nu=1}^{N(k, \tau)} \int_{\rho(x, y) \leq 2^{-k}} 2^{k}\left(2^{k \alpha} \sup _{z \in c Q_{\tau}^{k, \nu}}\left|D_{k}(f)(z)\right|\right)^{q} \chi_{Q_{\tau}^{k, \nu}}(x) d \mu(y)\right\}^{1 / q}\right\|_{p} \|_{p} \\
& \leq c \|\left\{\sum_{k \in \mathbb{Z}} \sum_{\tau \in I_{k}} \sum_{\nu=1}^{N(k, \tau)}\left(2^{k \alpha} \|_{z \in c Q_{\tau}^{k, \nu}}\left|D_{k}(f)(z)\right|\right)^{q} \chi_{Q_{\tau}^{k, \nu}}(x)\right\}_{p} \\
& \leq c\left\|\left\{\sum_{k \in \mathbb{Z}}\left(2^{k \alpha}\left|D_{k}(f)\right|\right)^{q}\right\}^{1 / q}\right\|_{p} .
\end{aligned}
$$

On the other hand, by Theorem 2, we have

$$
\begin{gathered}
\left\|\left\{\sum_{k \in \mathbb{Z}} \int_{\rho(x, y) \leq 2^{-k}} 2^{k}\left(2^{k \alpha}\left|D_{k}(f)(y)\right|\right)^{q} d \mu(y)\right\}^{1 / q}\right\|_{p} \\
=\left\|\left\{\sum_{k \in \mathbb{Z}} \sum_{\tau \in I_{k}} \sum_{\nu=1}^{N(k, \tau)} \int_{\rho(x, y) \leq 2^{-k}} 2^{k}\left(2^{k \alpha}\left|D_{k}(f)(y)\right|\right)^{q} \chi_{Q_{\tau}^{k, \nu}}(x) d \mu(y)\right\}^{1 / q}\right\|_{p}
\end{gathered}
$$




$$
\begin{aligned}
& \geq\left\|\left\{\sum_{k \in \mathbb{Z}} \sum_{\tau \in I_{k}} \sum_{\nu=1}^{N(k, \tau)} \int_{Q_{\tau}^{k, \nu}} 2^{k}\left(2^{k \alpha} \inf _{z \in Q_{\tau}^{k, \nu}}\left|D_{k}(f)(z)\right|\right)^{q} \chi_{Q_{\tau}^{k, \nu}}(x) d \mu(y)\right\}^{1 / q}\right\|_{p} \\
& \geq c\left\|\left\{\sum_{k \in \mathbb{Z}} \sum_{\tau \in I_{k}} \sum_{\nu=1}^{N(k, \tau)}\left(2^{k \alpha} \inf _{z \in Q_{\tau}^{k, \nu}}\left|D_{k}(f)(z)\right|\right)^{q} \chi_{Q_{\tau}^{k, \nu}}(x)\right\}^{1 / q}\right\|_{p} \\
& \geq c\left\|\left\{\sum_{k \in \mathbb{Z}}\left(2^{k \alpha}\left|D_{k}(f)\right|\right)^{q}\right\}^{1 / q}\right\|_{p} .
\end{aligned}
$$

Finally, Theorem 4 follows from Theorem 3 and a result in [H2].

\section{ACKNowledgement}

I would like to thank the referee for his comments.

\section{REFERENCES}

[Ch] M. Christ, The Tb theorem with remarks on analytic capacity and the Cauchy integral, preprint. MR 92k:42020

[CW] R. Coifman and G. Weiss, Analyse harmonique noncommutative sur certains espaces homogenes, Lecture Notes in Math., vol. 242, Springer-Verlag Berlin, Heidelberg, New York, 1971. MR 58:17690

[DJS] G. David, J.-L. Journe, and S. Semmes, Operateurs de Calderon-Zygmund, fonctions paraccretives et interpolation, Revista Mat. Iberoamericana, 1 (1985), 1-56. MR 88f:47024

[FJ] M. Frazier and B. Jawerth, A discrete transform and decompositions of distribution spaces, J. Funct. Anal., 93 (1990), 34-170. MR 92a:46042

[FS] C. Feferman and E. M. Stein, Some maximal inequalities, Amer. J. Math. 93 (1971), 107115. MR 44:2026

[H1] Y.-S. Han, Calderon-type reproducing formula and the Tb theorem, Revista Mat. Iberoamericana, vol. 10, (1994), 51-91. MR 95h:42020

[H2] , Discrete Calderon reproducing formula, preprint.

[HS] Y.-S. Han and E. T. Sawyer, Littlewood-Paley theory on spaces of homogeneous type and classical function spaces, Memoirs of the AMS 530 July 1994. MR 96a:42016

[M] Y. Meyer, Les nouveaux operateurs de Calderon-Zygmund, Asterisque, 131 (1985), 237254. MR 87f:42040b

[MS1] R. A. Macias and C. Segovia, Lipschitz functions on spaces of homogeneous type, Adv. in Math., 33 (1970), 257-270. MR 81c:32017a

[MS2] _ A decomposition into atoms of distributions on spaces of homogeneous type, ibid., 271-309.

Department of Mathematics, Auburn University, Auburn, Alabama 36849-5310

E-mail address: hanyong@mail.auburn.edu 